\title{
The labors of referees
}

\author{
Peer review is a cornerstone of the scientific publication process. High-quality refereeing is its essential element.
}

he evaluation of research studies by peers is central to the dissemination of robust, validated science. But no system is ever perfect, and neither is peer review. The seemingly straightforward process of soliciting the independent assessments of experts so that the editor may decide on whether to publish a particular study is in practice fraught with complexities, not least because it can be implemented in many different ways. Depending on the discipline, journal, editor and individual manuscript, all aspects of peer review can vary, from the number of referees, to the time taken to complete it, to the anonymity of authors and/or referees, the confidentiality or openness of the process, and the level of editorial oversight. More details on Nature Cancer's peer-review process can be found on our website and relevant Editorial ${ }^{1}$, but regardless of the different permutations of the peer-review process, one aspect remains a constant necessity: the quality of the refereeing. To guide Nature Cancer referees, we maintain a dedicated webpage for reviewers and provide further guidance when researchers accept the offer to review a manuscript.

We appreciate that refereeing is an additional laborious task in already hectic schedules. Thus, a prospective referee must consider carefully whether to accept an assignment, as the efficiency and consistency of the process relies on honoring time commitments and agreeing to review subsequent versions of the manuscript. If extending a deadline is necessary, it is always best to discuss this with the editor as early as possible, to ensure that the authors can receive a decision in a timely manner. Enlisting a knowledgeable lab member or collaborator to assist can be helpful, but it is essential to discuss this with the editor first and to ensure that confidentiality of the manuscript is maintained at all times. If a referee is unable to undertake an assignment, we welcome suggestions of other appropriate experts, as we are actively seeking to expand our referee populations by engaging a diverse group of researchers with respect to gender, geography, race/ ethnicity and career stage, as outlined in our diversity pledge.
Safeguarding the fairness of the process is also essential, and thus we ask prospective referees to recuse themselves if they have competing interests. Among these are work on closely overlapping topics, collaborations with the authors, positive or negative personal relationships, and potential effects of the reported findings at a professional or financial level that might compromise one's objectivity. Even when a reviewer can proceed in the absence of any such obvious conflicts, it is important to keep in mind that none of us are free of implicit biases. Thus the peer review of any given manuscript should be approached with a measure of self-awareness, with an open mind and by retaining the focus strictly on the reported science.

To that end, we ask referees to provide an objective and balanced assessment of the merits of the study, considering technical elements of experimental design and methodology, data quality, validity and reproducibility, analytical and statistical robustness, and the logic and reliability of conclusions. Beyond the technical side, we ask whether the reported findings constitute a significant addition to the published literature that would be of interest to the broad cancer community. The goal of peer review is to evaluate the suitability of a submission for this particular journal, so it is useful to keep in mind our scope and readership. An essential part of any referee report is guidance to improve the study through additional experiments. Such recommendations should center on experiments that would prove, strengthen or advance the findings in a manner directly relevant to the main message of the paper. It is best to avoid suggesting non-essential experiments that are peripheral or beyond the scope of the study under review. Comments on data and text presentation can be useful, but these should be restricted to major concerns about logic, clarity and accessibility, rather than minor linguistic or structural elements.

A great referee report is justified rather than superficial; balanced rather than dismissive or over-effusive; detailed but not consumed by minor points; specific but not unwieldy; intentional but grounded on feasibility; constructive even when recommending rejection; and always civil and measured in tone. A great referee adds value to the paper by helping the authors to improve it regardless of where it may ultimately be published.

When re-reviewing a manuscript, it is essential to comment on the revisions in response to the original concerns and to avoid raising new points on data already reviewed in previous rounds, unless a major issue was inadvertently missed. To streamline the re-review process, we discourage multiple major revision rounds and guide authors and referees closely on the points we consider essential for further consideration of the manuscript at our journal. We also stay in contact with both sides throughout the process, also notifying the reviewers of our decisions, revisions we consider priorities and points we have overruled, and seeking their additional input when necessary. Being a responsive and communicative referee is key to a smooth and effective peer-review process, be it about timelines, co-reviewing with others or about answering specific questions about the manuscript and comments.

Some consider refereeing a thankless but essential task; others are eager to participate in the peer-review and publication process; and yet others appreciate the benefits of gaining knowledge and seeing studies develop through critique. The Nature journals recognize the work of referees through referee activity reports and small tokens of appreciation in the form of personal journal subscriptions. We are keenly aware that these initiatives do not fully reflect the high-responsibility, time-consuming endeavor that is refereeing, and we are open to suggestions for improving referee recognition. We take this opportunity to express our deep gratitude to all the researchers who have contributed their time and expertise to the peer-review process at our journal.

Published online: 22 October 2021 https://doi.org/10.1038/s43018-021-00281-x

References

1. Nat. Cancer 2, 773-774 (2021). 